\title{
Ideas have Consequences: Eine vergleichende Analyse zur transformativen Rolle von Ideen
}

\author{
Christian Grimm*
}

\section{Zusammenfassung}

In diesem Beitrag wird die gesellschaftlich formende Rolle von Ideen am Beispiel von Friedrich August Hayeks Hegemonieansatz zur Durchsetzung einer neoliberal geprägten Weltanschauung thematisiert. In diesem Zusammenhang soll aufgezeigt werden, dass Hayek nicht der Einzige war, der sich diesem Thema zuwandte und an die Macht der Ideen glaubte. Dies rückt die Frage nach verwandten theoretischen Ansätzen ins Zentrum des Interesses. Konkret werden mit Ludwig Mises, John M. Keynes, Walter Lippmann und Antonio Gramsci vier Autoren aufgegriffen, deren Gedanken zur transformativen Rolle von Ideen hinsichtlich des Überschneidungsgrads mit dem Hayekschen Denken überprüft werden. Im Ergebnis wird ersichtlich, dass die genannten Autoren der Macht der Ideen einen hohen Stellenwert beimessen. So entwickelten diese Personen sehr ähnliche Theorien intellektueller Vorherrschaft, obgleich sie zum Teil sehr unterschiedlichen politischen Spektren entstammen.

Schlagwörter: Hegemoniegewinnung, Neoliberalismus, Hayek, Macht der Ideen

Ideas have Consequences: A comparative Analysis of the transformative Role of ideas

\section{Abstract}

Focus of this article is a discussion of the transformative role of ideas using the example of Friedrich August Hayek's hegemony theory for the implementation of a neoliberal dominated worldview. However, Hayek was not the only one, who believed in the power of ideas. Therefore, I discuss various forms of overlaps with other authors, namely Ludwig Mises, John M. Keynes, Walter Lippmann and Antonio Gramsci. The findings show, that these authors have developed quite similar theories in terms of intellectual supremacy, even though they come up from different political perspectives.

Keywords: Hegemony production, Neoliberalism, Hayek, Power of Ideas

\footnotetext{
* Christian Grimm, Trauner Verlag Linz, Institut für die Gesamtanalyse der Wirtschaft, Johannes Kepler Universität Linz. E-Mail:ch.grimm@gmx.at
} 


\section{Neoliberalismus und Hegemonie}

In kaum einer politischen Debatte, in der es um die Analyse sozioökonomischer Zustände oder Entwicklungen geht, wird nicht an einem bestimmten Punkt der Begriff des Neoliberalismus ins Feld geführt. Dieser ist zugleich auch Gegenstand kontroverser Auseinandersetzungen im akademischen Forschungsfeld. Auf beiden Ebenen entstammen die Beiträge einer kritischen Perspektive. Gerade im Kontext politischer Debatten wird der Begriff v. a. seit der Jahrtausendwende verstärkt pejorativ als „negativer Inbegriff des entfesselten, global agierenden Kapitalismus“ (Butterwegge et al. 2008: 14) verwendet. In diesem Zusammenhang wird auch konstatiert, dass das (westliche) wirtschaftspolitische System selbst nach der jüngsten Finanzkrise durch den Neoliberalismus geprägt wird (Aalbers 2013; Blyth 2013; Palley 2013; O’Donnell 2018). Diese Sichtweise wird nicht nur von den meisten KritikerInnen, sondern auch von BefürworterInnen wirtschaftsliberaler Konzeptionen vertreten, obgleich sich diese selbst nicht explizit als neoliberal bezeichnen (Mirowski 2014). So schreibt z. B. Gerhard Willke, dass sich "Neoliberalismus und Marktkapitalismus“ durchgesetzt haben, „weil sie besser sind als die (bislang) bekannten Systemalternativen“ (Willke 2003: 16). Auch in der österreichischen Parteipolitik gibt es seit den 1980er-Jahren einen zunehmenden Einfluss neoliberaler Argumentations- und Denkmuster (Grimm 2018), der unter anderem am Umbau des Sozialstaates zu beobachten ist (Rosenberger/Schmid 2003; Tálos 2005, 2006), wenngleich diese Position auch infrage gestellt wird (Zellenberg 2018). Im Kontext historischer und theoretischer Auseinandersetzungen überwiegen gleichfalls die kritischen Stimmen (Amadae 2003; Mirowski/Plehwe 2009; Mirowski 2013; Venugopal 2015), welche zuweilen auch sehr schroffe Züge annehmen (Schui et al. 1997; Harvey 2005; Saad-Filho/Johnston 2005).

Auch nach den jüngsten Krisenerscheinungen scheint das neoliberale Gedankengebäude, manche AutorInnen sprechen auch vom neoliberalen Finanzkapitalismus (Schulmeister 2013), über eine enorme Standfestigkeit $\mathrm{zu}$ verfügen. Ruft man sich die Anfänge seines nun knapp achtzigjährigen Bestehens in Erinnerung, ist dieser Umstand keineswegs als Selbstverständlichkeit zu sehen. ${ }^{1}$ Vielmehr begannen

1 Innerhalb des neoliberalen Gedankengebäudes muss freilich zwischen verschiedenen, teilweise sehr hete-
Anhänger des (Neo-)Liberalismus in einer Zeit, in der sich marktliberale Positionen in der Defensive befanden, an einer Strategie zur Hegemoniegewinnung zu arbeiten. Treibende Kraft hinter diesem Projekt war der österreichische Ökonom und Philosoph Friedrich August Hayek, der mit seinen Arbeiten zur spontanen Ordnung von Märkten (Hayek 1969a) nicht nur eine ganze ökonomische Denkschule prägte, sondern sich etwa in dem Aufsatz Intellectuals and Socialism (Hayek 1949) - auch intensiv mit Methoden zur Durchsetzung von Ideen und Meinungen befasste. Es ist daher mitunter das Verdienst von Hayek, dass das neoliberale Gedankengebäude in den 1970er-Jahren über eine gute institutionelle Basis verfügte und sich so als wirtschaftstheoretisches und -politisches Gegengewicht zum damals bestimmenden Keynesianismus profilieren konnte. Diese Tatsache führte in Kombination mit einer Reihe an realwirtschaftlichen Veränderungen (siehe nachstehend) zum neoliberalen Transformationsprozess in den westlichen Industrienationen (Overbeek 1993; Backhouse 2005; Kowall 2013).

Die Schwerpunktsetzung des vorliegenden Beitrags liegt auf einer hegemonietheoretischen Betrachtung der Durchsetzung des neoliberalen Gedankengebäudes. Demnach stehen nicht so sehr die ökonomischen Verhältnisse jener Zeit als eher die erdachten Techniken zur organisierten intellektuellen Einflussnahme im Zentrum des Interesses. Dahinter steht die These, dass die Persistenz der gegenwärtig vorherrschenden neoliberalen Spielart des Kapitalismus durch eine erfolgreiche Umgestaltung des gesellschaftlichen Meinungsklimas (Hegemoniegewinnung) befördert wurde. $^{2}$ Diese Aussage darf jedoch nicht dahin missverstanden werden, als dass sämtliche wirtschaftliche

\footnotetext{
rogenen Schulen differenziert werden, die dennoch durch gewisse Leitgedanken verbunden sind (siehe: Kapitel 2). Diese Bandbreite und Wandlungsfähigkeit ist zweifelsohne als eine Stärke des Neoliberalismus zu werten. Kompakte Darstellungen über die verschiedenen Formen des Neoliberalismus finden sich beispielsweise bei Plehwe/Walpen (1999) oder bei Walpen (2000).

2 Unter Hegemonie wird in Anschluss an Antonio Gramsci ein Machttypus bezeichnet, „der im Wesentlichen auf der Fähigkeit basiert, eigene Interessen als gesellschaftliche Allgemeininteressen zu definieren und durchzusetzen“" (Brand/Scherrer 2003: 92). Hegemonie ist ein Verfahren, bei dem die herrschende Klasse auf mittelbaren Zwängen die Zustimmung der Bevölkerung zu ihrer Herrschaft zu gewinnen versucht. Sie ist dann erreicht, wenn die breite Masse glaubt, dass die Entscheidungen der herrschenden Klasse im allgemeinen Interesse sind.
} 
Erscheinungen auf einen umfassenden Masterplan zurückzuführen sind, welcher vonseiten des marktliberalen Akteurs-Netzwerkes erdacht, überwacht und dirigiert wird. Das wäre trügerisch. Treffender ist die Sichtweise des Bestrebens neoliberaler DenkerInnen, auf Basis der Verbreitung von Wissen die öffentliche Meinung in eine bestimmte Richtung treiben zu können. In diesem Zusammenhang relevant ist der oben erwähnte Aufsatz von Hayek, in dem er die strategische Bedeutung von Ideen zur Verwirklichung wirtschaftspolitischer Vorstellungen hervorhebt und die Formulierung eines „liberal Utopia“ (Hayek 1949: 432) anregt. Aber nicht nur Hayek ist von der Rolle der Ideen als Katalysator zur gesellschaftlichen Transformation überzeugt. In einigen Punkten finden sich Berührungspunkte mit den Schriften anderer Autoren, die sowohl aus dem eigenen als auch aus anderen politischen Lagern kommen (Mukand/Rodrik 2018). Aus methodischer Sicht entspricht dieser Artikel einer Theoriearbeit, in welcher der Frage nach etwaigen Inspirationsquellen für das Hayeksche Denken nachgegangen wird. Hierbei wird bewusst auf zum Teil sehr unterschiedliche Theorietraditionen Bezug genommen (liberal, reformistisch, marxistisch). Konkret soll nachgezeichnet werden, welche Berührungspunkte hinsichtlich der Macht der Ideen bzw. Intellektuellen im Vergleich zu Ludwig Mises, John M. Keynes, Walter Lippmann und Antonio Gramsci identifiziert werden können. Die Forschungsarbeit orientiert sich in erster Linie an Primärquellen, um eine möglichst getreue Wiedergabe der einzelnen Positionen zum Zweck des Abgleichs von Verwandtschaftsbeziehungen sicherzustellen. Übergeordnetes Ziel dieses Beitrags ist es also, die Veränderung der gesellschaftlichen, politischen und ökonomischen Landschaft als eine Veränderung des (intellektuellen) Denkstils begreiflich zu machen (Polanyi-Levitt 2014). Dabei soll nicht nur auf die Kraft der Ideen und Meinungen innerhalb des neoliberalen Spektrums hingewiesen, sondern darüber hinaus auch ein Bewusstsein dafür generiert werden, dass gegensätzliche politische Lager ähnliche Theorien intellektueller Vorherrschaft entwickelt haben.

Die weitere Untersuchung ist wie folgt gegliedert: Zunächst wird in aller Kürze eine historische Einordnung des Neoliberalismus vorgenommen, wobei in erster Linie auf die ideengeschichtliche Situation rund um die Krise des klassischen Wirtschaftsliberalismus als Ursprung der neoliberalen Bewegung eingegangen wird (Kapitel 2). Danach werden mit Mises, Keynes, Lippmann und Gramsci vier Autoren aufgegriffen, die als Impulsgeber für Hayeks Theorie zur Hegemoniegewinnung betrachtet werden können (Kapitel 3). Im Anschluss daran wird Hayeks eigene strategische Herangehensweise behandelt, wobei neben seinen theoretischen Überlegungen (Elitentheorie) auch die Umsetzung in die Praxis (Organisation) diskutiert wird (Kapitel 4). Im Resümee werden die Positionen der vier betrachteten Autoren hinsichtlich der Berührungspunkte mit Hayeks Denken verglichen (Kapitel 5).

\section{Zur Genese des Neoliberalismus}

Ökonomische Theorien entstehen häufig vor dem Hintergrund konkreter Wirtschaftsprobleme. Dies trifft beispielsweise auf Keynes' General Theory of Employment, Interest and Money (1936/2009) zu, die als Reaktion auf die Rekordarbeitslosigkeit zur Zeit der Großen Depression zu verstehen ist, oder auf Nicholas Georgescu-Roegens Entropy Law and the Economic Process (1971), das die irreversible Entwertung der Natur infolge der auf Massenproduktion und -konsumption ausgerichteten (keynesianischen) Marktwirtschaft in den Nachkriegsjahrzehnten thematisierte. Ähnlich verhält es sich beim Neoliberalismus, dessen Gerüst vor dem Hintergrund der Weltwirtschaftskrise ab 1929 und der Abwendung vieler politischer und ökonomischer Entscheidungsträger von liberalen Ideen erwuchs (Burgin 2012). An dieser Stelle ist es nicht zielführend, die Geschichte des Neoliberalismus umfassend nachzuzeichnen (hierzu siehe z. B.: Walpen 2004; Nordmann 2005; Thomasberger 2012). Vielmehr werden v. a. jene ideen- und wirtschaftsgeschichtlichen Aspekte beleuchtet, welche die Genese dieses vielschichtigen Gedankengebäudes bis hin zur Gründung der Mont Pèlerin Society (MPS) 1947 mitbeeinflusst haben.

Der (Wirtschafts-)Liberalismus sah sich bereits Ende des 19. Jahrhunderts mit einer Reihe verschiedener Herausforderungen konfrontiert. ${ }^{3}$ Infolge der umfassenden Industrialisierung in Verbindung mit einem raschen Bevölkerungsanstieg in West- und Zentraleuropa wurden neue Formen sozialer Missstände sichtbar. So wurde die Verelendung großer Teile der lohnarbeitenden Bevölkerungsschicht in politischen

3 Diese sind nicht gesondert voneinander zu sehen, sondern bedingen sich gegenseitig. So wird beispielsweise die fundamentale Frage nach der ökonomischen Organisation einer modernen Gesellschaft durch die einschneidenden Finanz- und Wirtschaftskrisen 1873 und 1929 immer wieder neu befeuert. 
Debatten als soziale Frage thematisiert (Wendt 2016: 110). Die Pauperisierung stellte aber nicht nur den damals verbreiteten Glauben an den Fortschritt in der Gesellschaft in Frage, sondern führte darüber hinaus - betreffend der Frage nach der wirtschaftlichen Organisation einer modernen Gesellschaft - zu einer scharfen Auseinandersetzung zwischen AnhängerInnen verschiedener ideologischer Strömungen. Hierbei übten sozialreformerische, aber auch radikalsozialistische Theorien mit ihrem Bestreben der Transformation der bürgerlich-kapitalistischen Gesellschaft eine zunehmend stärkere Anziehungskraft aus (Sykes 1997/2014; Walpen 2000). Im Zuge der russischen Oktoberrevolution 1917 und der Gründung der UdSSR wurde diese Frage um eine realpolitische Dimension erweitert. Liberale Parteien sahen sich jedoch nicht nur Angriffen von sozialistischer, sondern auch von nationalistisch-protektionistischer Seite ausgesetzt. So wurde beispielsweise in Deutschland 1873 der Verein für Socialpolitik als interventionistischer Gegenpart zu den wirtschaftsliberalen Vorstellungen des Kongresses Deutscher Volkswirte gegründet (Hirte 2019: 394f.). Beide Strömungen - sozialistisch wie nationalistisch - räumen dem Staat eine aktivere Rolle bei der Ausgestaltung des Wirtschaftsprozesses ein. Beiden ist eine antiliberale Haltung gemeinsam. ${ }^{4}$

Der antiliberale Zeitgeist wurde innerhalb des neoliberalen Spektrums erstmals von Mises pointiert in Gemeinwirtschaft zum Ausdruck gebracht. Mises bezeichnete den Sozialismus als „die Losung unserer Tage“ (Mises 1922/1932: 1). Und weiter:

„Die sozialistische Idee beherrscht heute die Geister.

Ihr hängen die Massen an, sie erfüllt das Denken und Empfinden aller, sie gibt der Zeit ihren Stil. Die Geschichte wird über den Abschnitt, in dem sie von uns berichtet, die Worte setzen: das Zeitalter des Sozialismus. (...) Einige Völker sind daran gegangen, das sozialistische Programm mit einem Schlage bis in seine letzten Auswirkungen durchzuführen. Vor unseren Augen hat der russische Bolschewismus ein Werk vollbracht, das, (...) schon wegen der Großartigkeit seines Entwurfes zu dem Merkwürdigsten gerechnet werden muss, das die Weltgeschichte gesehen hat." (ebd.: 1)

$4 \quad$ Unter den Neoliberalen betont insbesondere Hayek die Ähnlichkeiten der Anschauungen von Sozialismus und Nationalismus, die er im gemeinsamen Feind in Gestalt des Liberalismus geeint sieht (Hayek 1944/2014: 47ff., 210ff., $227 \mathrm{ff}$.).
Neben den grundsätzlichen Diskussionen über die ökonomische Organisationsweise wurde das Vertrauen in den Wirtschaftsliberalismus auch infolge mehrerer einschneidender Finanz- und Wirtschaftskrisen stark in Mitleidenschaft gezogen. Innerhalb von nur knapp 50 Jahren erlebten die westlichen Industriestaaten zwei Börsenzusammenbrüche, den Gründerkrach 1873 und die Weltwirtschaftskrise 1929 (Senft 2010; Pressler 2013). $\mathrm{Zu}$ jener Zeit waren wirtschaftsliberale Vorstellungen bei einem Großteil der Intellektuellen aus Wirtschaft und Politik, aber auch in der breiten Öffentlichkeit weitgehend diskreditiert. Im Gegensatz dazu erlebten - wie vorhin schon erwähnt - politisch extreme Ideologien (z. B. Kommunismus in der UdSSR, Faschismus im Königreich Italien, Nationalsozialismus im Dritten Reich) starken Zulauf, aber auch interventionistische - im Einklang mit Keynes' General Theory stehende Konzepte wurden zum Credo staatlicher Wirtschaftspolitik (z. B. New Deal in den USA; Schweden).

Die Genese des Neoliberalismus ist vor dem Hintergrund der Krise des klassischen (Wirtschafts-) Liberalismus zu sehen. Bereits in den 1930er-Jahren bemühte sich eine kleine, aber international agierende Gruppe, eine neue Variante des Wirtschaftsliberalismus in Abgrenzung zum Laissez-faire-Prinzip des 19. Jahrhunderts aufzubauen. ${ }^{5}$ Aus dem zunächst nur losen Kontakt zwischen einzelnen Anhängern begann sich allmählich ein neues liberales Netzwerk zu formieren. Hayek fungierte dabei als ein zentraler Angelpunkt, unterhielt er doch Kontakte zur Freiburger Schule (Walter Eucken), zur London School of Economics (LSE) (Lionel Robbins) sowie zum französischen Philosophen Louis Rougier. Letzterer war Mitbegründer des Verlags Librairie de Médicis, in welchem wichtige Werke neoliberaler Gelehrter zur Beeinflussung der wirtschaftspolitischen Diskussion in Frankreich publiziert wurden (Denord 2001: 18ff.). Daneben fungierte Rougier auch als Initiator des ersten internationalen Treffens neoliberaler Intellektueller. Dieses fand 1938 in Paris statt (Colloquium Walter Lippmann), wo

5 Walter Lippmann sah im Laissez-faire-Prinzip den „Kardinalfehler des Liberalismus des 19. Jahrhunderts“ (Lippmann 1937/1945: 249). Zu einer ähnlichen Einschätzung gelangte auch Hayek in seinem viel rezipierten Werk Weg zur Knechtschaft: „Nichts dürfte der Sache des Liberalismus so sehr geschadet haben wie das starre Festhalten einiger seiner Anhänger an gewissen groben Faustregeln, v. a. an dem Prinzip des Laissez-faire“ (Hayek 1944/2014: 37). Aus der Reihe der Ordoliberalen schrieb z. B. Wilhelm Röpke vom „Grundirrtum des Laissez-faire-Prinzips“" (Röpke 1942/1979: 394). 
neben einer breiten Diskussion über die Gründe des Niedergangs des Liberalismus und den Bedingungen seiner Rückkehr auch der Begriff Neoliberalismus als (positive) Selbstbezeichnung für die neue Wirtschaftskonzeption eingeführt wurde (Walpen 2000: 1071). ${ }^{6}$ In dieser ersten Phase ging es aber v. a. darum, die vereinzelten Anhänger neoliberaler Anschauungen zusammenzuführen - sie wird daher auch als „Verteidigungs- bzw. Konsolidierungsphase“ (Plehwe/Walpen 1999: 20) bezeichnet.

Die Bewegungsphase des neoliberalen Netzwerkes setzte erst nach dem Zweiten Weltkrieg ein, als Hayek im April 1947 eine zweite internationale Konferenz im schweizerischen Mont Pèlerin arrangierte, zu dessen Gründungstreffen sich 39 Intellektuelle, vorwiegend Ökonomen und Philosophen, einfanden. Als kurze gemeinsame programmatische Grundlage der MPS wurde von Robbins das Statement of Aims ausgearbeitet, das die geschickt gewählte Formulierung einer „redefinition of the functions of the state " enthält (MPS online). Dabei ging es darum, die Rolle des Staates in einer liberalen Marktwirtschaft neu zu definieren, weil die alte Vorstellung des Laissez-faire-Liberalismus spätestens mit der Great Depression als gescheitert angesehen werden musste. Dieser neuen Konzeption zufolge soll der Staat keinesfalls abgebaut oder zerschlagen werden, sondern durch bewusstes Eingreifen in die Wirtschaft einen liberalen Interventionismus zur Stärkung des Marktes oder, um es mit Hayek auszu-

6 Wenngleich für dieses Unterfangen ein gemeinsamer Terminus technicus gefunden wurde, welcher später auch durch Robbins Dokument einen schriftlichen Minimalkonsens erhielt, darf nicht übersehen werden, dass das neoliberale Gedankengebäude und folglich auch die MPS über eine enorme inhaltliche Bandbreite an Denkschulen (z. B. Österreichische Schule, Freiburger Schule, Chicago School) verfügt. Auch auf personeller Ebene existierten zum Teil starke interne Differenzen. Bekannt ist in diesem Zusammenhang Alexander Rüstows Brief an Röpke, in dem er anregt, Mises und Hayek als Paläoliberale in Spiritus gesetzt ins Museum zu stellen (Walpen 2000: 1076). Ungeachtet dessen gibt es in der Wirkungsgeschichte des Neoliberalismus einige zentrale Leitgedanken, die als theoretische Bindeglieder zwischen den Denkschulen fungieren. Hierunter fällt $u$. a. die Ablehnung kollektivistischer Strömungen, welche Sozialismus/Kommunismus und Nationalsozialismus, aber auch Keynesianismus/Interventionismus und Wohlfahrtsstaat inkludieren. Ferner gilt der Wettbewerbsmarkt als hauptsächliche Koordinationsinstanz für wirtschaftliche Tätigkeiten. In diesem Zusammenhang wird es als staatliche Aufgabe gesehen, die Durchsetzung und Sicherung der marktwirtschaftlichen Funktionsfähigkeit zu gewährleisten (liberaler Interventionismus) (Grimm 2018: 140). drücken, eine Planung zum Zwecke des Wettbewerbs betreiben (Hayek 1944/2014: 66). Mit dieser speziellen Wortwahl verdeutlicht Hayek den Unterschied zwischen einer liberalen und einer totalitären Wirtschaftsordnung (Kollektivismen). Da aus seiner Sicht letztere den gesellschaftlichen Diskurs beherrschen, entwickelte er einen hegemonialen Ansatz, um das öffentliche Meinungsklima wieder zugunsten (neo-)liberaler Anschauungen zu verändern. Im Zuge der internen Diskussionen setzte sich Hayeks Position gegen andere Sichtweisen durch (Plehwe/Walpen 1999). Seine Strategie, das „Klima der Ideen“ zu beeinflussen, weist dabei zahlreiche Parallelen mit Theorien anderer Autoren aus zum Teil völlig verschiedenen politischen Spektren auf.

\section{Ideen und Intellektuelle: Hayeks Anknüpfungspunkte}

Bevor Hayeks eigene hegemonietheoretische Herangehensweise zur Verbreitung marktliberaler Ideologie thematisiert wird, erfolgt zunächst ein Rückgriff auf eine Reihe sehr unterschiedlicher Denker, deren Gemeinsamkeit u. a. in dem Standpunkt zu finden ist, dass die politische Entwicklung stark von der Verbreitung bestimmter Ideen abhängig ist.

\subsection{Ludwig Mises}

Ein erster Anknüpfungspunkt findet sich in der Person Ludwig Mises, dessen Wirkung auf Hayek nicht hoch genug eingeschätzt werden kann. Mises' Denken stellt in der Wirkungsgeschichte des Neoliberalismus ein zentrales Fundament dar (Walpen 2000: 1069). Zum einen setzte er sich bereits in den 1920er-Jahren kritisch mit dem Sozialismus auseinander und nahm hierbei zentrale neoliberale Argumentationsmuster vorweg, die auch in späteren Werken Widerhall fanden (Mises 1922/1932, 1929/2013). ${ }^{7}$ Überdies hielt er im Roten Wien der Zwischenkriegszeit in seinem Handelskammerbüro regelmäßig abendliche Privatseminare ab, in denen er sich als entschiedener Gegner sozialistischer Ideen positionierte (Haberler 1981). Zu den Teilnehmern zählte neben Fritz

7 Mises sieht im Sozialismus ein destruktives Element: „Der Sozialismus ist in Wahrheit nicht das, was er $\mathrm{zu}$ sein vorgibt. Er ist nicht Wegbereiter einer besseren und schöneren Zukunft, sondern Zertrümmerer dessen, was Jahrtausende der Kultur mühsam geschaffen haben." (Mises 1922/1932: 424) Er schlägt bei seiner Kritik am Sozialismus insofern ähnlich schroffe Töne an, wie sie heute teilweise von GegnerInnen des Neoliberalismus verwendet werden. 
Machlup, Gottfried Haberler und Oskar Morgenstern auch der junge Hayek, der unter Mises' Einfluss seine Sympathien für die Ideen der Fabianischen Sozialisten ablegte und sich zu einem überzeugten Anhänger der Marktwirtschaft entwickelte (Klein 2013). Nicht nur deswegen stellte die Begegnung mit Mises für Hayek ein dauerhaft prägendes Schlüsselereignis dar.

In Bezug auf Hayeks hegemonialen Ansatz ist v. a. Mises' Praxeologie, womit er die Lehre von allem menschlichen Handeln bezeichnet, von Interesse. Mises betrachtet das gesellschaftliche Zusammenwirken in einer modernen (Industrie-)Gesellschaft als einen komplexen, von Komplikationen und Unübersichtlichkeiten gekennzeichneten Zustand. Folglich kann es keine historischen Gesetzmäßigkeiten oder geschichtlichen Automatismen geben, die den Gang der gesamtgesellschaftlichen Entwicklung erklären können:

„Theorie vom menschlichen Handeln und Geschichte stehen in unüberbrückbarem logischen Gegensatz. Die Theorie kann nur apriorisch sein; die Geschichte kann sich immer nur des individuellen Verhaltens bedienen und kann niemals aus ihrem Erfahrungsmaterial empirische Gesetze gewinnen. (...) Die Versuche, historische Gesetze aufzustellen, sind so vollkommen gescheitert, dass es sich nicht lohnt, sich mit ihnen näher zu befassen. "(Mises 1940: 48f.)

Nichtsdestoweniger basiert jedwedes gesellschaftliche Zusammenwirken auf einer gewissen Ordnung, welche vor ihrer Ausführung zuerst erdacht werden muss. Diese Ordnung entsteht nach Mises nicht durch einen vollständig ausgearbeiteten (gesamtwirtschaftlichen) Plan, sondern ist eine Konsequenz des ökonomischen Handelns von Einzelpersonen. Beispielsweise müssen zwei Individuen, die untereinander eine Tauschbeziehung durchführen wollen, diese zunächst in Gedanken durchspielen. Aus ökonomischer Perspektive ist die Gesellschaft ein positives, nichtintendiertes Ergebnis der individuellen Interaktionen. Zugleich sieht er menschliche Handlungsweisen in einer bestehenden Gesellschaftsordnung als ein Produkt der Ideologie. Mises konstruiert eine klare kausale Abhängigkeit zwischen Idee und Handlung: ${ }^{8}$

„Die Gesellschaft ist das Erzeugnis menschlichen Handelns. Menschliches Handeln wird von den Ideologien bestimmt. Mithin ist Gesellschaft ein Produkt der Ideologie,

8 Mises erörtert das menschliche Bewusstsein somit aus zwei Blickwinkeln: Während aus der ökonomischen Perspektive Faktoren wie Knappheit und Nutzen das menschliche Handeln leiten, sind es auf der zweiten Ebene die vorherrschenden Ideen. und nicht die Ideologie ein Produkt der Gesellschaft." (ebd.: 166)

Der Schlüssel zur Veränderung einer Gesellschaft ist für Mises also weniger in objektiven Tatbeständen als in der Ideenwelt zu finden - es ist das Bewusstsein, das das Sein bestimmt. Dauerhaft gesicherte gesellschaftliche Machtverhältnisse bedürfen eines ideologischen Fundaments. In Die Gemeinwirtschaft schreibt Mises dem Sozialismus den Status der ideologischen Vorherrschaft zu. Dabei betont er schon damals die Rolle der geistigen Führer, da sie es sind, die den Ideen des Sozialismus anhängen und ihre Gedanken unter den Massen verbreiten. Als Konsequenz dessen ist Mises bestrebt, die Intellektuellen vom Sozialismus loszulösen und sie für den (Neo-)Liberalismus zu gewinnen. Auf diese Weise würde ihnen - so hoffte er - automatisch auch die breite Masse der Bevölkerung folgen:

„Wenn die Intelligenz sich vom Sozialismus abwenden würde, dann wäre es um die Macht des Sozialismus geschehen. Die Massen können auf die Dauer den Ideen der Führer keinen Widerstand entgegenstellen." (Mises 1922/1932: 472)

Hayek schließt an Mises' Gedanken gleich in mehrerlei Hinsicht an. Zum Ersten spricht er sich konsequent gegen alle Ansätze aus, Gesellschaft, Wirtschaft und Kultur als Ergebnis zielgerichteter menschlicher Planung zu begreifen. Für Hayek ist jeder Versuch einer gesamtwirtschaftlichen Systemplanung unüberwindbaren Grenzen ausgesetzt (hierzu siehe z. B.: Hayek 1945, 1988, 1996). Zum Zweiten übernimmt Hayek die Position des Primats der Ideen als treibende Kraft für die gesellschaftliche Entwicklung und das damit einhergehende indeterminierte Geschichtsverständnis. Demzufolge kann die ideologische Vormachtstellung nur im Wettbewerb der Ideen erreicht werden. Hier findet sich schließlich eine dritte Gemeinsamkeit. Beide sehen das Grundproblem darin, dass sich die Intellektuellen der falschen Ideologie (Sozialismus) zugehörig fühlen, weswegen Hayek bei der Gründungstagung der MPS auch darauf insistierte, nur gleich gesinnte Denker aufzunehmen, um eine liberale Gegenhegemonie zu errichten (Walpen 2004: 104).

\subsection{John Maynard Keynes}

Mises mag das Denken von Hayek insbesondere in dessen jungen Jahren maßgeblich geprägt haben, doch war er bei Weitem nicht die einzige Inspirationsquelle. Hayek griff für sein Hegemoniekonzept auch auf Autoren zurück, die seiner ökonomischen Gegenseite zuzurechnen sind. Hierbei ist v. a. Keynes zu nennen, 
der im letzten Kapitel seiner General Theory die Wirkmächtigkeit intellektueller Vordenker und deren Ideen zur Beeinflussung politischer, wirtschaftlicher oder gesellschaftlicher Entwicklungen herausstrich (Keynes 1936/2009).

Das Verhältnis zwischen Hayek und Keynes ist ambivalent. Hayek wurde 1931 von Robbins für vier Gastvorlesungen an die LSE eingeladen. Bereits ein Jahr darauf erhielt er dort als erster nicht britischer Staatsbürger eine Professur (Economics and Statistics). Hayek fungierte dabei auch als marktliberales Gegengewicht zu den interventionistischen Sichtweisen von Keynes und seinen Anhängern an der Cambridge University (Cockett 1995). In jener Zeit lieferten sich beide Ökonomen einen wissenschaftlichen Schlagabtausch. Bekannt ist in diesem Zusammenhang eine in den Jahren 1931 und 1932 geführte Debatte, die u. a. in der Zeitschrift Economica ausgetragen wurde. ${ }^{9}$ Ungeachtet der vorhandenen Differenzen in ökonomischen Sachfragen, pflegten beide jedoch einen achtungsvollen, ja sogar freundschaftlichen Umgang miteinander (Skidelsky 1992: 459). Insbesondere als die LSE zwischen 1940 und 1944 kriegsbedingt nach Cambridge verlegt wurde, bestand zwischen ihnen ein reger Kontakt, den Hayek später als sehr angenehm darstellte:

„Diejenigen unter uns, die das Glück hatten, ihn persönlich zu treffen, verspürten bald die Anziehungskraft des brillanten Gesprächspartners mit seinen weiten Interessen und seiner bezaubernden Stimme." (Hayek 1969b: 91)

Vier Jahre nach dieser theoretischen Debatte wurde die General Theory veröffentlicht, welche $\mathrm{zu}$ einem Wendepunkt im ökonomischen Denken führte. Hayeks Reaktion darauf ist in doppelter Hinsicht interessant: Zum einen blieben die von Keynes aufgestellten theoretischen Aussagen und Zusammenhänge zunächst weitgehend unkommentiert, obgleich in seinen späteren Werken zahlreiche Hinweise auf Kritik zu finden sind (Sanz Bas 2011). Zum anderen, weil gerade die sozialphilosophischen Schlussbetrachtungen in der General Theory bei Hayek einen prägenden Eindruck hinterlassen haben. Als sich 1947 das Gründungstreffen der MPS zutrug, befand sich der (Wirtschafts-)Liberalismus in einer veritablen Krise, was Hayek dazu veranlasste, eine

9 Hayek setzte sich zu Beginn kritisch mit Keynes' A Treatise on Money auseinander und unterstellte ihm hierbei eine unzureichende kapitaltheoretische Fundierung (Hayek 1931, 1932), woraufhin dieser mit einer teils harschen Replik auf Hayeks Preise und Produktion konterte (Keynes 1931) konterte.
Strategie für dessen Neuerrichtung zu entwerfen. Dabei verwies er explizit auf die Gedanken von Keynes, der von einer nachhaltigen Gestaltungsfunktion der Ideen ökonomischer und sozialphilosophischer Intellektueller ausging:

„Praktiker, die sich ganz frei von intellektuellen Einflüssen glauben, sind gewöhnlich die Sklaven irgendeines verblichenen Ökonomen. Verrückte in hoher Stellung, die Stimmen in der Luft hören, zapfen ihren wilden Irrsinn aus dem, was irgendein akademischer Schreiberling ein paar Jahre vorher verfasste. Ich bin überzeugt, dass die Macht erworbener Rechte im Vergleich zum allmählichen Durchdringen von Ideen stark übertrieben wird. " (Keynes 1936/2009: 323)

Keynes nahm dabei an, dass Ideen nicht unmittelbar, sondern erst über einen längeren Zeitraum von 25 oder 30 Jahren wirksam werden. Im Einklang mit Keynes' Aussagen stehend, gelangte Hayek zu der Schlussfolgerung, dass es zur Verwirklichung einer neoliberalen Weltanschauung der Zusammenführung gleichgesinnter DenkerInnen bedarf, weswegen er wie vorhin erwähnt - die MPS als eine nach außen hin geschlossene Vereinigung entstehen ließ. Nur durch die Entwicklung einer einheitlichen Ideenbasis lässt sich in der Gesellschaft eine neue liberale Weltanschauung etablieren. Dies ist jedoch in keinem kurzfristigen Projekt realisierbar, vielmehr bedarf es eines beständigen Engagements, wie Hayek selbst vermerkte:

„Die öffentliche Meinung ist das Werk von unseresgleichen, von Nationalökonomen und Wirtschaftsphilosophen vergangener Generationen, die die politische Atmosphäre geschaffen haben, in der sich die Politiker unserer Zeit bewegen müssen. (...) In dieser langen Sicht müssen wir unsere Aufgabe betrachten. Wir müssen uns mit den Meinungen befassen, die sich verbreiten müssen, wenn eine freie Gesellschaft erhalten oder wiederhergestellt werden soll, nicht mit dem, was im Augenblick durchführbar erscheint. " (Hayek 1947/1952: 142f.)

\subsection{Walter Lippmann}

Während Keynes' sozialphilosophische Überlegungen zur Rolle von Ideen in seinem ökonomischen Hauptwerk nur eine kleine Randbemerkung ausmachen, beschäftigt sich Walter Lippmann in seinem zentralen Werk The Good Society viel ausführlicher mit der Frage der Durchsetzung von (neoliberalen) Ideen. Er geht dabei weit über Keynes hinaus, da er auch Wege zur langfristigen Umgestaltung von vorhandenen Meinungen bespricht. Lippmann stützt sich dabei auf seine prakti- 
schen Erfahrungen aus der Zeit des Ersten Weltkriegs, als er als Mitglied des Committee on Public Information daran beteiligt war, die US-amerikanische Bevölkerung vom Kriegseintritt zu überzeugen (Trudel 2017: 3722). Lippmann nahm in den 1930er-Jahren eine bedeutende Rolle innerhalb der neoliberalen Bewegung ein. The Good Society verschaffte ihm innerhalb dieses Spektrums große Beachtung (Walpen 2004: 55). ${ }^{10}$ Auf Geheiß Röpkes wurde es 1945 unter dem Titel Gesellschaft freier Menschen ins Deutsche übersetzt. ${ }^{11}$ Bereits 1938 berief Rougier aufgrund von Lippmanns Erfolg das nach ihm benannte Colloquium ein, um die Hauptthesen aus seinem Werk zu diskutieren (Reinhoudt/Audier 2018). Bei diesem Treffen war auch Hayek anwesend, dessen politisches Denken durch Lippmanns Schrift merklich geformt wurde, was nicht zuletzt an den Parallelen zwischen The Good Society und Der Weg zur Knechtschaft augenscheinlich wird (Jackson 2012).

Doch schon 15 Jahre bevor The Good Society erschien, schrieb Lippmann ein Werk, in dem er sich mit der Frage nach den Techniken zur Beeinflussung der breiten Masse beschäftigte und das bis heute als wichtiges Werk in der Stereotypenforschung gilt. Ausgangspunkt ist der Gedanke, dass zwischen Mensch und äußerer Handlungswelt (Realität) eine „konstruierte“ Welt (Pseudoumwelt) liegt, die aus mentalen Bildern und Stereotypen besteht, welche das Handeln der Menschen in der realen Welt bestimmen (Lippmann 1922/1964: 64). In der Folge befasst sich Lippmann mit der Frage, wie die (politische) Meinungsbildung durch imaginative Bilder gestaltet werden kann. Hierbei übernehmen mentale Bilder bzw. Stereotypen eine entscheidende Funktion - sie vereinfachen komplexe Zusammenhänge aus der realen Welt und sind als Orientierung in derselben auch ein notwendiges Element. Nach Lippmann ist die dadurch konstruierte Pseudoumwelt aber keine individuelle, sondern eine gemeinsame Welt:

„Die Bilder in den Köpfen dieser menschlichen Wesen, die Bilder von sich selbst, von anderen, von ihren Bedürfnissen, Zielen und Beziehungen zueinander sind ihre öffentlichen Meinungen. Diejenigen Bilder, nach denen ganze Gruppen von Menschen oder Individuen im Namen von Gruppen handeln, sind die öffentliche Meinung."

(Lippmann 1922/1964: 28)

Lippmann beschreibt auch in Ansätzen, wie mentale Bilder vermittelt werden können und differenziert hierbei zwischen zwei Kategorien von Intellektuellen: Autoritäten versorgen die Menschen mit Informationen. Sie gelten als vertrauenswürdig und stehen an der Spitze von Institutionen, sodass die Bevölkerung einfach mit ihnen in Berührung kommt. Der Bogen dessen, wen Lippmann als Autorität sieht, ist sehr weit gespannt. Im Wesentlichen setzt er aber auf traditionelle Hierarchien, wie z. B. Parteiführer, Priester, Grundbesitzer, Industrielle, Könige. Die zweite Gruppe stellen Fachmänner mit einem jeweils hoch spezialisierten Wissensgebiet dar. Diese sind jedoch nicht imstande, die öffentliche Meinung derart stark zu beeinflussen wie die Autoritäten, da die Masse mit ihnen kaum in Berührung gerät (ebd.: 156ff.).

Auch The Good Society handelt von der Frage der aktiven Beeinflussung des gesellschaftlichen Denkens - eine Fragestellung, die von Lippmann in den Kontext der Konfrontation zwischen (Neo-)Liberalismus und Kollektivismus eingebettet wird. Damit ist er auf inhaltlicher und strategischer Ebene ein Vorläufer der hegemonietheoretischen Überlegungen von Hayek. Lippmanns Werk ist zweigeteilt. Teil eins liefert eine harsche Kritik an kollektivistischen Strömungen, worunter er sowohl Kommunismus als auch Faschismus subsumiert. Diesen autoritären Systemen wird in Teil zwei die Freiheit des Liberalismus gegenübergestellt (Lippmann 1937/1945). Für dessen Wiederkehr ist ein Wandel des Denkens notwendig. Dazu müssen die in der Öffentlichkeit vorhandenen mentalen Bilder umgestaltet werden. Lippmann geht wie Hayek von einer gesellschaftlichen Diskurshoheit des Kollektivismus aus. Mit der Umgestaltung der mentalen Bilder wird nicht so sehr darauf abgezielt, das praktische Handeln der Menschen unmittelbar zu verändern, sondern den Diskurs auf der Ebene der Pseudoumwelt zu formen und so Hegemonie herzustellen (ebd.: 165). Auch Lippmann sieht den Erfolg der Auswirkungen eines Wandels im Denken nur über eine lange Zeitspanne gegeben. Konkret veranschlagt er für diese Unternehmung 75 Jahre (ebd.: 91, 491): ${ }^{12}$

12 Lippmann berief sich bei seinen Überlegungen ebenso wie später dann Hayek auf Keynes: „Im Reich der Ideen wirkt sich der Wandel einer Theorie erst nach einiger Zeit in der Praxis aus und wenden Praktiker einer Epoche, wie Keynes sagt, im allgemeinen die Theorie längst verstorbener Männer an.“ (Lippmann 1937/1945: 91) Sprache ein. 
„Wenn ich sage, dass die Wiedergeburt des Liberalismus gewiss ist, so meine ich damit natürlich nicht, dass sie noch in unserer Zeit kommen muss. "(ebd.: 280)

Die Parallelen zwischen Lippmann und Hayek erstrecken sich über Inhalt, Ziel und Methodik. Beide sehen im Kollektivismus/Sozialismus als dominante Weltanschauung die Hauptproblematik ihrer Zeit. Dementsprechend verfolgten sie die Mission der Erneuerung des Liberalismus. An diesem Punkt nahm Lippmann die spätere strategische Herangehensweise zur Überwindung der Krise des Liberalismus von Hayek vorweg, indem er ein auf Hegemoniegewinnung ausgerichtetes Projekt umriss. Allerdings wichen sie in ihren Schwerpunktsetzungen etwas voneinander ab. Lippmanns Hauptaugenmerk lag auf der Frage, wie sich Meinungen verbreiten lassen (mentale Bilder), jenes von Hayek, an wen sie vermittelt werden und wie diese Vermittlung organisiert sein muss (siehe: Kapitel 4).

\subsection{Antonio Gramsci}

Neben den drei bisher behandelten Autoren weisen auch die Schriften des italienischen Marxisten Antonio Gramsci Parallelen mit Hayeks hegemonietheoretischen Überlegungen auf, was angesichts der Tatsache, dass Hayek und Gramsci völlig gegensätzlichen politischen Lagern zuzuordnen sind und sich beide auch persönlich nie begegneten, ein bemerkenswerter Umstand ist. Sowohl Gramsci als auch Hayek entwickelten ein strategisches Konzept zur Erlangung und Erhaltung von Hegemonie, bei dem die Intellektuellen eine bedeutende Rolle einnehmen. Gramsci wird daher in dieser Auflistung als Letztes behandelt, wenngleich seine Schriften im Zuge seiner politischen Gefangennahme durch die italienischen Faschisten bereits Ende der 1920er- und Anfang der 1930er-Jahre und somit vor Keynes' General Theory und Lippmanns Good Society entstanden.

Kernfrage ist für Gramsci jene nach der inneren Stabilität in kapitalistischen Gesellschaften. Während Russland durch die Oktoberrevolution $\mathrm{zu}$ einem kommunistischen Staat transformiert wurde, setzte in Zentral- und Westeuropa kein derartiger Systemwandel ein. Gramsci rückt bei seiner Erklärung die Machtausübung auf der Ebene der Zivilgesellschaft in den Vordergrund und entwirft in diesem Kontext das Konzept der kulturellen Hegemonie. Er differenziert hierbei den integralen Staat in zwei Gesellschaftsbereiche mit verschiedenen Praxen der Machtaus- übung. ${ }^{13}$ Die politische Gesellschaft ist gleichbedeutend mit dem eigentlichen Staatsapparat, worunter die Gesamtheit der politischen und juristischen Strukturen wie Gerichte, Regierung, Polizei und Armee zu verstehen ist. Als zivile Gesellschaft sind jene Institutionen gemeint, die das kulturelle, soziale und religiöse Zusammenleben der Menschen regeln. Hierzu zählen Medien, Familie, Kirche, Verbände und Parteien (Gramsci 1992b). Während in der politischen Sphäre (Staatsapparat) Macht durch Herrschaft in Form von Gewalt und Zwang zum Ausdruck kommt, wird Macht in der Sphäre der Zivilgesellschaft über Hegemonie errungen. Hegemonie entspricht einer (bestimmten) geistigen, kulturellen und politischen Führung der Gesellschaft, wobei die Regierenden um die Zustimmung der Regierten bemüht sind (Neubert 2001: 66). Sie ist dabei im Sinne eines Prozesses immer wieder zu organisieren und gegen äußere Angriffe zu verteidigen. Gramsci sieht eine entwickelte Zivilgesellschaft als ausschlaggebendes Charakteristikum für kapitalistische Systeme und deren Stabilität an, da die Massen durch Teilhaberechte inkludiert und so an die herrschenden Verhältnisse gebunden werden. Der integrale Staat mit seinem Wechselspiel aus politischer und ziviler Gesellschaft erklärt für Gramsci das Scheitern kommunistischer Revolutionen in kapitalistischen Regimen:

„Im Osten war der Staat alles, die Zivilgesellschaft war in ihren Anfängen und gallertenhaft; im Westen bestand zwischen Staat und Zivilgesellschaft ein richtiges Verhältnis, und beim Wanken des Staates gewahrte man sogleich eine robuste Struktur der Zivilgesellschaft. Der Staat war nur ein vorgeschobener Schützengraben, hinter welchem sich eine robuste Kette von Festungen und Kasematten befand, von Staat zu Staat mehr oder weniger, versteht sich [...]“"(Gramsci 1992b: 874).

Im Folgenden widmet Gramsci sich der Frage, wie Hegemonie erlangt und verteidigt werden kann. Die Durchsetzung von Ideen soll dabei von Intellektuellen herbeigeführt werden. Gramsci unterscheidet in seiner Hegemonietheorie zwischen organischen und traditionellen Intellektuellen. Die Gruppe der organischen Intellektuellen wird von jeder gesellschaftlichen Klasse aus ihren eigenen Reihen hervorgebracht. Für Gramsci gibt es somit keine gesamtgesellschaftlich homogene Intellektuellengruppe. Vielmehr erfül-

13 Er weist jedoch darauf hin, dass die Trennung aus rein methodischer Sicht erfolgt, da in der gesellschaftlichen Praxis beide Kategorien als Einheit zu sehen sind (Gramsci 1992a: 498). 
len die organischen Intellektuellen die Aufgabe der Selbstverständigung innerhalb ihrer jeweiligen Klassen, indem sie ein Bewusstsein der eigenen Funktion generieren. Zugleich versuchen sie spezielle Meinungen und Normen auszuarbeiten und gegenüber anderen Klassen zu behaupten (Gramsci 1996: 1497). Die zu dieser Gruppe zählenden Intellektuellen (z. B. Schriftsteller, Lehrer, Ärzte, Ingenieure) verfügen in ihrem Umfeld über Prestige und Glaubwürdigkeit und sorgen als „Ideenmultiplikator“ dafür, dass die herrschenden Verhältnisse akzeptiert werden. Letztendlich produzieren sie gewissermaßen Hegemonie. Die Gruppe der traditionellen Intellektuellen inkludiert schließlich jene Personen, die in der Gesellschaft historisch gewachsene Tätigkeiten $-\mathrm{z}$. B. im religiösen Bereich oder im Bildungswesen - ausüben und dabei im Laufe der Zeit ein gewisses Maß an Autonomie gegenüber der herrschenden politischen Klasse erlangen konnten.

Das dynamische Element bei der Ausgestaltung des gesellschaftlichen Meinungsklimas liegt bei den organischen Intellektuellen. Eine soziale Klasse, welche eine neue Hegemonie etablieren will, hat zuerst ihre eigene organische Gruppe herauszubilden. Mit ihr muss sie versuchen, die traditionellen Intellektuellen für ihre eigenen Ansichten zu gewinnen (ebd.: 1500). Ferner muss die nach der Macht greifende Klasse bereit sein, Abstriche bei ihren Interessen zu machen und mit anderen politischen Kräften Allianzen zu bilden. Die Kompromisse stoßen dort an ihre Grenzen, wo sie die ökonomischen Grundlagen einer Klasse in Gestalt ihrer gesellschaftlichen Lage in den Produktionsverhältnissen gefährden. Insofern ist die Änderung des Bewusstseins bei Gramsci auch mit der materiellen Basis verbunden.

Gramscis Überlegungen kommen jenen von Hayek in vielen Punkten sehr nahe. Entscheidend ist die gemeinsame Einsicht, dass die Errichtung von Hegemonie nicht auf Basis verordneter staatlicher Zwangsmaßnahmen, sondern nur in einem Kampf um die Köpfe erfolgen kann. Gewonnen werden kann dieser nur, wenn es gelingt, das gesellschaftliche Kräfteverhältnis durch intellektuelle Einflussnahme entsprechend den Sichtweisen der nach dem Einfluss strebenden Gruppe anzupassen. Hier findet sich zugleich ein wesentlicher Unterschied: Hayek geht (wie auch Mises und Lippmann) von einer primär ideengeleiteten Veränderung aus, Gramsci bezieht hierbei, auch aufgrund seines marxistischen Hintergrunds, die materielle Ebene stärker ein.

\section{Die Durchsetzung des Neoliberalismus: Hayeks Strategie zur Hegemoniegewinnung}

Das folgende Kapitel widmet sich der organisatorischen Ausgestaltung des neoliberalen Projektes, wobei neben der Netzwerkformierung v. a. die theoretischen Überlegungen von Hayek, die zu eben jener praktischen Umsetzung geführt haben, skizziert werden. Die Auseinandersetzung mit Hayeks Theorie dient dabei weniger der Beweisführung, dass Hayeks Position jahrzehntelang die strategische Ausrichtung der MPS bestimmt hat (hierzu siehe z. B.: Plehwe/Walpen 1999; Walpen 2004), sondern wird vorrangig mit dem Ziel einer vergleichenden Darstellung Hayeks mit den bereits genannten Denkern vorgenommen. Daher wird von der umfassenden Hayek-Literatur auch nur jener kleine Teilbereich miteinbezogen, mit dem Hayek die strategische Ausrichtung der MPS vorgab und der die Beeinflussung des Meinungsklimas zum Gegenstand hat. Wie schon seine „Vordenker“ glaubte Hayek an das Prinzip: „Ideas have Consequences.“

In The Intellectuals and Socialism entwickelt er eine Strategie zur organisatorischen Verbreitung neoliberaler Ideen am erfolgreichen „Modell“ der Durchsetzung des Sozialismus. Das Grundproblem liegt für Hayek darin, dass die Fundamente einer freien Gesellschaft von sozialistischen Intellektuellen untergraben werden (Hayek 1949: 429). Die Etablierung des Sozialismus als hegemoniales Element in Politik und Gesellschaft habe sich nur deswegen zutragen können, da „socialist ideals governed the thinking of the more active intellectuals" (ebd.: 417). Folglich wendet sich Hayek den Intellektuellen zu, mit dem Ziel, langfristig die öffentliche Meinung zugunsten des Neoliberalismus zu verändern. Auch er nimmt in seiner Theorie eine Aufteilung der Intellektuellen in zwei verschiedene Kategorien vor und unterscheidet zwischen original thinkers und secondhand dealers in ideas. Die original thinkers generieren als innovative Genies ersten Ranges wesentliche Grundkonzepte und stehen daher an der Spitze des Meinungsbildungsprozesses. ${ }^{14}$ Dennoch stellen sie nicht das zentrale Element in seiner Theorie dar, weil die Entwicklung von Ideen erst durch die organisatorische Vermittlung gesellschaftlich in einem hegemonialen Sinne wirksam werden kann. Genau jene Funktion wird von den secondhand dealers übernommen. Diese Personen (z. B. Journalisten, Lehrer, Schriftsteller,

14 Für Hayek ist dabei der Philosoph der Prinz unter den Intellektuellen (ebd.: 423). 
Künstler, Ärzte) zeichnen sich dadurch aus, dass sie mit vielen verschiedenen Themen in Berührung kommen und letztendlich darüber entscheiden, welche Ideen in die Gesellschaft transferiert werden: ${ }^{15}$

"It is the intellectuals in this sense who decide what views and opinions are to reach $u$, which facts are important enough to be told to us and in what form and from what angle they are to be presented. Whether we shall ever learn of the results of the work of the expert and the original thinker depends mainly on their decision. "(ebd.: 419)

Am unteren Ende steht die breite Masse der Bevölkerung, bei der sich die vermittelten Ideen quasi automatisch festsetzen (ebd.: 421). Insofern gelten sie als manipulierbare Größe, deren revolutionäres Potenzial allerdings für die herrschende Klasse stets eine gewisse Bedrohung darstellt (Plehwe/Walpen 1999: 211). Der Informationsfluss bei der Ideenvermittlung folgt nach Hayek somit einer hierarchischen Struktur, wenngleich zwischen den zwei Kategorien von Intellektuellen Abhängigkeiten existieren.

Hayek führt die Stärke des Sozialismus in erster Linie darauf zurück, dass dessen VertreterInnen es geschafft haben, allgemeine Ideen mit visionärem Charakter zu entwickeln, während sich die Anhänger des Liberalismus zu sehr in Detailfragen verloren hätten:

„In particular, socialist thought owes its appeal to the young largely to its visionary character; the very courage to indulge in Utopian thought is in this respect a source of strength to the socialists which traditional liberalism sadly lacks. (...) Once the basic demands of the liberal programs seemed satisfied, the liberal thinkers turned to problems of detail and tended to neglect the development of the general philosophy of liberalism. "(Hayek 1949: 428)

Als Konsequenz dessen ist von den Neoliberalen eine eigene Utopie zu erarbeiten, wofür es - analog der Vorgehensweise der Sozialisten - der Entwicklung allgemeiner Ideen bedarf. Diese sollen nicht $\mathrm{zu}$ sehr tagespolitische Problemfelder bedienen oder an den Möglichkeiten der momentanen Umsetzung ausgerichtet sein, als mehr den Charakter einer abstrakten Vision besitzen. Auf diese Weise sind sie bei den intellektuellen Vermittlern durchsetzungsfähig, da sich diese nach Hayeks Auffassung gedanklich weniger mit

15 Hayek beschreibt diese Gruppe trotz ihres maßgeblichen Einflusses auf die öffentliche Meinung abwertend, da sie weder besondere Fähigkeiten benötigen, noch von den Ideen, die sie verbreiten, viel verstehen müssen. Sie sind nur "masters of the technique of conveying ideas" (ebd.: 418).
Detailfragen beschäftigen, sondern vagen Konzepten den Vorzug geben (ebd.: 432). Hayek geht davon aus, dass die Erneuerung des (Neo-)Liberalismus ein langfristiges Unterfangen ist und dieser erst in zwei bis drei Generationen den Status einer hegemonialen Philosophie erlangen kann (ebd.: 424).

Neben seinen theoretischen Überlegungen lag Hayeks Fokus v. a. auf der praktischen Strategie der Wissensvermittlung zur Gewinnung von Hegemonie. Insofern war er sehr bemüht, die materielle Infrastruktur zur organisierten Ideenverbreitung aufzubauen. Auf diesem Wege entstanden verschiedene Kanäle der Einflussnahme, die bei der Propagierung neoliberaler Inhalte ein Schlüsselelement einnahmen. Zum einen gelang es, Allianzen mit gleichgesinnten DenkerInnen, u. a. aus dem Medien- und Journalismuswesen, herzustellen, die mit ihren Artikeln und Kommentaren den (Alltags-)Diskurs in der wirtschaftsliberalen Publizistik beeinflussten. Besonders enge personelle Verbindungen gab es beispielsweise zur Neuen Zürcher Zeitung, wo in den 1950er- und 6oer-Jahren auch einige Artikel zu den ansonsten geschlossenen MPS-Konferenzen veröffentlicht wurden (Plickert 2008). Darüber hinaus wurde auch versucht, gezielt im Bereich der Wissenschaft zu intervenieren. Hierzu wurden ab den 1940er-Jahren diverse wirtschaftsliberale Zeitschriften (z. B. ORDO 1948, The Freeman 1950, Faith and Freedom 1950, National Review 1955, New Individualist Review 1961) ins Leben gerufen (Nash 1976: 27f; Walpen 2004: 73, 92).

Den höchsten Stellenwert nahm für Hayek allerdings die Bildung eines Think-Tank-Netzwerkes zur langfristigen Errichtung einer neoliberalen Hegemonie ein. Die bedeutendste Vereinigung und zugleich das Ideenzentrum dieses Netzes ist bis heute die von ihm 1947 gegründete MPS (siehe z. B. Hartwell 1995). ${ }^{16}$ Ihre Funktion liegt nach George Stigler, Nobelpreisträger und langjährigem MPS-Mitglied, aber weniger in der unmittelbaren Beeinflussung politischer Entscheidungen:

"It publishes no books or periodicals, engages in no political acitivity, issues no proclamations, and its members continue to embrace a fairly wide spectrum of attitudes on economic and political affairs." (Stigler 1988: 146)

Treffender ist daher die Bezeichnung als formlose Arbeits- und Diskussionsplattform, die im Hayekschen Sinne als die Organisation der original thinkers für

16 Neben der MPS hatte Hayek auch an der Neugründung der Chicago School of Economics sowie an der Gründung des Institute of Economic Affairs wesentlichen Anteil. 
Austausch- und Vernetzungsmöglichkeiten angelegt ist.

Als Mitte der 1970er-Jahre das wirtschaftspolitische System des keynesianischen Fordismus mit einer Reihe an Problemen zu kämpfen hatte (Stagflation, Auflösung des Bretton-Woods-Systems, Konflikte zwischen Arbeit und Kapital, Ressourcenknappheit infolge von Massenproduktion), traten die neoliberalen Akteure auf den Plan, um das entstandene Machtvakuum mit eigenen Lösungsvorschlägen und Reformkonzepten zu füllen. Der neoliberale Transformationsprozess auf wirtschaftspolitischer Ebene setzte somit tatsächlich erst rund drei Jahrzehnte später ein, nachdem strategisch damit begonnen wurde, eine Veränderung des öffentlichen Meinungsklimas herbeizuführen. Die Renaissance des Liberalismus war kein terminiertes Ereignis und noch weniger wurde sie von ihren führenden Vertretern anhand eines durchdachten Plans heraufbeschworen. Noch in den 196oer-Jahren - zur Hochzeit des Keynesianismus - entsprachen neoliberale Standpunkte einer Minderheitenposition mit einem verschwindend geringen Einfluss auf die wirtschaftliche Praxis (Kowall 2013). Abgesehen von ein paar Intellektuellen aus Wissenschaft, Politik und Medien konnte in der öffentlichen Meinung keine neoliberale Hegemonie errichtet werden, da selbst in eher konservativen Kreisen das Prinzip einer Allianz zwischen Unternehmern und Arbeiterschaft aufrechterhalten wurde (Rothschild 2009). Zu jener Zeit war der Gedanke eines umfassenden neoliberalen Transformationsprozesses also abwegig, auch wenn - wie in der BRD - während der 1950er-Jahre die wirtschaftspolitische Umsetzung des Ordoliberalismus in Gestalt der sozialen Marktwirtschaft vorangetrieben wurde (Hagemann 2017). Umgekehrt hätte die neoliberale Wende ohne die immensen intellektuellen Vorleistungen rund um das von Hayek initiierte Think-Tank-Netzwerk der MPS mit größter Wahrscheinlichkeit in dieser Form nicht stattgefunden.

\section{Resümee: Die transformative Macht der Ideen}

In diesem Beitrag wurde die gesellschaftlich formende Rolle von Ideen am Beispiel der Durchsetzung des neoliberalen Gedankengebäudes thematisiert. Historischer Ausgangspunkt war die Krise des (Wirtschafts-) Liberalismus, die in der Zeit der Great Depression ihren Höhepunkt erreichte, zugleich aber den Beginn der neoliberalen Formierungsphase markierte. In diesem Kontext geläufig ist der von Hayek formulierte hege- moniale Ansatz zur Neubelebung liberaler Werte. Obgleich seine strategische Rolle bei der Entfaltung von Diskursmacht nicht hoch genug einzuschätzen ist, war er nicht der Einzige, der sich diesem Thema zugewendet hat. So sind manche seiner Überlegungen bereits bei Vertretern aus den eigenen Reihen zu finden. Weniger bekannt ist die Tatsache, dass auch Ähnlichkeiten mit diversen Autoren existieren, die ökonomisch aus dem reformistischen bzw. marxistischen Lager stammen. Es war daher das Bestreben, in diesem Beitrag mit Mises, Keynes, Lippmann und Gramsci zumindest vier Autoren zu besprechen und nach deren Berührungspunkten mit dem Hayekschen Denken zu suchen. Während Mises, Keynes und Lippmann als unmittelbare Inspirationsquellen bezeichnet werden können, ist dies bei Gramsci jedoch umstritten - ungeachtet der tatsächlichen Parallelen in ihren Theorien. Abgesehen von den vier hier behandelten Autoren lassen sich allerdings noch weitere mögliche Vordenker (z. B. Rougier, William Rappard) identifizieren (Walpen 2004: 56), insofern besitzt diese Betrachtung keinen erschöpfenden Charakter.

Im Ergebnis kann zunächst festgehalten werden, dass die hier untersuchten Autoren durch den prinzipiellen Standpunkt geeint sind, dass Ideen eine transformative Rolle in der Gesellschaft spielen. Einer Änderung der gesellschaftlichen, politischen und ökonomischen Situation muss zuerst eine Änderung des Bewusstseins vorausgehen. Einvernehmlich wird von den hier betrachteten Autoren auch auf die langfristige Perspektive hingewiesen, die ein solcher Wandel mit sich bringt. Bemerkenswerterweise entwickelten dabei Personen aus unterschiedlichen politischen Spektren ähnliche Theorien intellektueller Vorherrschaft, wobei sie jeweils aus dem Status einer vermeintlichen Marginalisierung heraus argumentierten. Dies ist auch deswegen interessant, weil die für die Analyse konstitutiven Werke in einer relativ kurzen Zeitspanne, nämlich zwischen den 1920er- und 1940er-Jahren, verfasst wurden. Während Gramsci die Gründe für das Scheitern kommunistischer Revolutionen in Zentralund Westeuropa suchte, gingen Hayek, Mises und auch Lippmann davon aus, dass die bestehende Gesellschaft gerade von kollektivistischen Ideologien à la Sozialismus, Faschismus und Keynesianismus beherrscht wird. Ausgehend von diesen Ansichten versuchen alle Seiten die Marginalität der eigenen Denkrichtung zu überwinden. Dies trifft auch auf Keynes' Intention zu, wenngleich sein Schwerpunkt auf einem Wandel im ökonomischen Denken lag. 
Wiewohl bei allen vier Autoren Gemeinsamkeiten mit Hayeks Ansichten zur transformativen Rolle von Ideen festzustellen sind, ist doch der jeweilige Grad der Überschneidungen verschieden stark ausgeprägt. Auch wenn sich Hayek beim MPS-Gründungstreffen explizit auf Keynes' sozialphilosophischen Schlussteil bezog, weisen die Ausarbeitungen der beiden nur oberflächliche Parallelen auf. Zwar unterstreicht Keynes die Bedeutung der Intellektuellen (Ökonomen und Philosophen), deren Ideen eine nachhaltige Gestaltungsfunktion ausüben, doch findet sich bei ihm weder eine ausgearbeitete Beeinflussungsstrategie zur organisatorischen Vermittlung oder Verbreitung von Meinungen noch zielen seine Überlegungen darauf ab, eine bestimmte Klasse als Hegemon zu installieren.

Anders bei Mises, der aus seiner Analyse die Konsequenz zieht, die geistigen Führer vom sozialistischen Gedankengut abzubringen, um so dessen dominante Stellung in der Öffentlichkeit zu brechen. Mises' theoretische Ausgangslage ist somit vergleichbar mit jener von Hayek. Auch verfolgen beide die gleiche Intention in Bezug auf die Wiedereinsetzung einer liberalen Weltanschauung. Mises beschränkt sich aber weitgehend auf eine Gegenüberstellung der für ihn zwei zentralen Wirtschaftsformen und bleibt eine genaue Strategie der liberalen Rückeroberung der Eliten zur Überwindung des Sozialismus schuldig. Ohne Frage wird bei ihm die meinungsformende Rolle der Intellektuellen auf die breite Masse klar erkannt, die letztlich wie bei Hayek als manipulierbare Größe dargestellt wird. Im Unterschied zu Hayek nimmt Mises allerdings keine funktionale Differenzierung zwischen Intellektuellen vor.

Die theoretischen Ausarbeitungen von Gramsci weisen eine Reihe an Parallelen zu jenen Hayeks auf. Beide beschäftigen sich mit der Frage, wie Wissen in der Gesellschaft wirksam werden kann, und entwickeln hierfür eine kompakte Hegemonieanalyse. Dies geschieht jedoch in einem jeweils gänzlich anderen historischen Kontext. Gramscis Vorstellung war es, dass aus der Klasse der Arbeiterschaft eine Hegemoniestrategie erwachsen würde, Hayek strebte hingegen eine neoliberal dominierte Weltanschauung an. Beide Autoren sahen sich somit aufgrund der vermeintlichen Dominanz des jeweils anderen politischen Lagers in der Defensivposition und hofften entsprechend, dass die eigene Seite die Hegemonie erlangen kann. Wie Hayek so schenkt auch Gramsci den Intellektuellen bei der Gewinnung von Hegemonie besondere Aufmerksamkeit. Auch er unterscheidet zwischen zwei Kategorien, sein Intellektuellenbegriff ist aber deutlich weiter gefasst, da er davon ausgeht, dass jede Klasse ihre eigenen (organischen) Intellektuellen hervorbringt. Diese entstammen folglich nicht nur dem Bürgertum, sondern auch der Arbeiterklasse. In ihrer Funktion als „Ideenmultiplikator" entsprechen Gramscis organische Intellektuelle Hayeks secondhand dealers. Für Gramsci ist eine Änderung des Bewusstseins allerdings auch mit der materiellen Basis verknüpft, sodass die Beeinflussung der öffentlichen Meinung nicht so weitreichend möglich ist, wie dies bei Hayek der Fall ist, der ausschließlich bei der Verbreitung von Ideen ansetzt.

Das Gedankengebäude von Lippmann kommt jenem von Hayek womöglich am nächsten. Beide sind sich in Ausgangspunkt (Dominanz der Kollektivismen) und Zielsetzung (Renaissance des Liberalismus) einig. Vor diesem Hintergrund beschäftigen sie sich - ähnlich zu Gramsci - mit der gleichen Ausgangsfrage, nämlich wie eine bestimmte Form des Wissens in einer Gesellschaft zur Herrschaftsdoktrin werden kann. Beide Autoren unterscheiden im Rahmen ihrer Theorie zwischen zwei Kategorien von Intellektuellen. Diese werden zwar unterschiedlich betitelt, gleichen sich aber im Wesentlichen hinsichtlich ihrer Funktionen. Die Fachmänner bzw. original thinkers entwickeln die Ideen, die von den Autoritäten bzw. secondhand dealers in die Öffentlichkeit transferiert werden. Hayek und Lippmann teilen somit den Fokus auf Institutionen als Teil des unpersönlichen Mechanismus bei der Ideenverbreitung. So können Lippmanns Autoritäten die Menschen gerade deswegen so gut mit Informationen versorgen, weil sie an der Spitze von Institutionen stehen. Hayek schätzt die secondhand dealers (nur) deswegen, da sie Meister der Ideenvermittlung sind. Als weitere Gemeinsamkeit klingt bei beiden Ansätzen eine elitäre Prägung durch, da die Beeinflussungsstrategie entlang einer Hierarchie verläuft. In zumindest einem Punkt weichen die beiden jedoch etwas voneinander ab: Im Unterschied zu Lippmann konzentriert sich Hayek mehr auf die Frage nach der organisatorischen Vermittlung von Meinungen, da die Intellektuellen dies nicht nur kraft ihrer gesellschaftlichen Position vermögen, sondern v. a. weil sie die Techniken der Vermittlung beherrschen.

Im Zuge der hier angestellten Überlegungen wurde gezeigt, dass die Macht der Ideen zur Erlangung von Hegemonie von Vertretern völlig unterschiedlicher weltanschaulicher Traditionen hoch bewertet wird. 


\section{Literatur}

Aalbers, M. B. (2013): Neoliberalism is Dead ... Long Live Neoliberalism! International Journal of Urban and Regional Research, 37 (3), 1083-1090.

Amadae, S. M. (2003): Rationalizing Capitalist Democracy: The Cold War Origins of Rational Choice Liberalism. Chicago: University of Chicago Press.

Backhouse, R. (2005): The Rise of Free Market Economics: Economists and the Role of the State since 1970. History of Political Economy, 37 (5), 355-392.

Brand, U./Scherrer C. (2003): Contested Global Governance: Konkurrierende Formen und Inhalte globaler Regulierung. Kurswechsel. Zeitschrift für gesellschafts-, wirtschafts- und umweltpolitische Alternativen, 1, 90-103.

Blyth, M. (2013): Austerity: The History of a Dangerous Idea. New York: Oxford University Press.

Burgin, A. (2012): The Great Persuasion. Reinventing the Free Market since the Depression. Cambridge, MA: Harvard University Press.

Butterwegge, C./Lösch, B./Ptak, R. (2008): Kritik des Neoliberalismus. Wiesbaden: VS Verlag für Sozialwissenschaften.

Cockett, R. (1995): Thinking the Unthinkable. Think-Tanks and the Economic Counter-Revolution 1931-1983. London: Harper Collins Publishers.

Denord, F. (2001): The Origins of Neoliberalism in France. Louis Rougier and the 1938 Walter Lippmann Conference. Le Mouvement Social, 195, 9-34.

Georgescu-Roegen, N. (1971): The Entropy Law and the Economic Process. Cambridge, MA: Harvard University Press.

Gramsci, A. (1992a): Gefängnishefte. Kritische Gesamtausgabe. Band 3, Hefte 4-5. Hamburg: Argument Verlag.

Gramsci, A. (1992b): Gefängnishefte. Kritische Gesamtausgabe. Band 4, Hefte 6-7. Hamburg: Argument Verlag.

Gramsci, A. (1996): Gefängnishefte. Kritische Gesamtausgabe. Band 7, Hefte 12-15. Hamburg: Argument Verlag.

Gramsci, A. (1998): Gefängnishefte. Kritische Gesamtausgabe. Band 8, Hefte 16-21. Hamburg: Argument Verlag.

Grimm, C. (2018):Wirtschaftspolitische Positionen österreichischer Parteien im historischen Verlauf. Die Ausgestaltung österreichischer Parteiprogrammatiken hinsichtlich neoliberalen Gedankenguts. Momentum Quarterly, 7 (3), 136-154.

Haberler, G. (1981): Reminiscences. Mises' Private Seminar. Wirtschaftspolitische Blätter, 28 (4), 121-126.

Hagemann, H. (2017): Ordoliberalism, the Social-Market Economy, and Keynesianism in Germany, 1945-1974. In: Backhouse, R. E./Bateman, B. W./ Nishizawa, T./Plehwe, D. (Hg.): Liberalism and the Welfare State: Economists and Arguments for the Welfare State. Oxford: Oxford University Press, 57-74.

Hartwell, M. (1995): A History of the Mont Pèlerin Society. Indianapolis: Liberty Fund.

Harvey, D. (2005): A Brief History of Neoliberalism. New York: Oxford University Press.
Hayek, F. A. (1931): Reflections on the Pure Theory of Money of Mr. J. M. Keynes. Economica, 33, 270-295.

Hayek, F. A. (1932): Reflections on the Pure Theory of Money of Mr. J. M. Keynes (continued). Economica, 35, 22-44.

Hayek, F. A (1944 [2014]): Der Weg zur Knechtschaft. Reinbek: Lau Verlag.

Hayek, F. A. (1945): The Use of Knowledge in Society. American Economic Review, 35 (4), 519-530.

Hayek, F. A. (1947 [1952]): „Freie Wirtschaft“ und Wettbewerbsordnung. In: derselbe: Individualismus und wirtschaftliche Ordnung. Erlenbach-Zürich: Rentsch, 141-155.

Hayek, F. A. (1949): The Intellectuals and Socialism. University of Chicago Law Review, 16 (3), 417-433.

Hayek, F. A. (1969a): Der Wettbewerb als Entdeckungsverfahren. In: derselbe: Freiburger Studien. Gesammelte Aufsätze von Friedrich August Hayek. Tübingen: Mohr-Siebeck, 249-265.

Hayek, F. A. (1969b): Persönliche Erinnerungen an Keynes und die „Keynessche Revolution“. In: derselbe: Freiburger Studien, Tübingen: Mohr-Siebeck, 90-96.

Hayek, F. A. (1988): Die verhängnisvolle Anmaßung. Die Irrtümer des Sozialismus. Tübingen: Mohr-Siebeck.

Hayek, F. A. (1996): Die Anmaßung von Wissen. Tübingen: Mohr-Siebeck.

Hirte, K. (2019): Die deutsche Agrarpolitik und Agrarökonomik: Entstehung und Wandel zweier ambivalenter Disziplinen. Wiesbaden: Springer.

Jackson, B. (2012): Freedom, the common good, and the rule of law: Lippmann and Hayek on economic. Journal of the History of Ideas, 73 (1), 47-68.

Keynes, J. M. (1931): The Pure Theory of Money. A Reply to Dr. Hayek. Economica, 34, 387-397.

Keynes, J. M. (1936 [2009]): Allgemeine Theorie der Beschäftigung, des Zinses und des Geldes. Berlin: Duncker \& Humblot.

Klein, D. B. (2013): Friedrich A. Hayek. Econ Journal Watch 10 (3), 352-358.

Kowall, N. (2013): Das neoliberale Modell. Genese, Politiken, Bilanz. Eine polit- und makroökonomische Bestandsaufnahme. Materialien zu Wirtschaft und Gesellschaft, Nr. 124. Kammer für Arbeiter und Angestellte für Wien. Online: https://emedien.arbeiterkammer.at/viewer/!fulltext/ AC11206616/2/ [24.03.2019].

Lippmann, W. (1922 [1964]): Die öffentliche Meinung. München: Rütten \& Loening.

Lippmann, W. (1937 [1945]): Die Gesellschaft freier Menschen. Bern: Verlag A. Francke.

Mirowski, P./Plehwe, D. (2009, Hg.): The Road from Mont Pelerin. The Making of the Neoliberal Thought Collective. Cambridge, MA: Harvard University Press.

Mirowski, P. (2013): Never Let a Serious Crisis go to Waste. How Neoliberalism Survived the Financial Meltdown. New York/London: Verso.

Mirowski, P. (2014): The Political Movement that Dared not Speak its own Name. The Neoliberal Thought Collective 
Under Erasure. Institute for New Economic Thinking Discussion Paper 14/23, New York.

Mises, L. (1922 [1932]): Die Gemeinwirtschaft. Untersuchungen über den Sozialismus. Jena: Verlag Gustav Fischer.

Mises, L. (1929 [2013]): Kritik des Interventionismus. Untersuchungen zur Wirtschaftspolitik und Wirtschaftsideologie der Gegenwart. München: H. Akston Verlag.

Mises, L. (1940): Nationalökonomie. Theorie des Handelns und Wirtschaftens. Genf: Editions Unions.

Mont Pèlerin Society (2019): Statement of Aims. Online: https://www.montpelerin.org/statement-of-aims/ [28.03.2019].

Mukand, S./Rodrik, D. (2018): The Political Economy of Ideas: On Ideas Versus Interests in Policymaking. NBER Working Papers No. 24467.

Nash, G. H. (1976): The Conservative Intellectual Movement in America. Since 1945. New York: Basic Books.

Neubert, H. (2001): Antonio Gramsci: Hegemonie - Zivilgesellschaft - Partei. Hamburg: VSA.

Nordmann, J. (2005): Der lange Marsch zum Neoliberalismus. Vom Roten Wien zum freien Markt - Popper und Hayek im Diskurs. Hamburg: VSA.

O'Donnell, A. (2018): Neoliberalism, Ambiguity and the Rise of Populist Movements. International Journal of Social Economics, 45 (7), 1030-1041.

Overbeek, H. (1993): Restructuring hegemony in the global political economy: The rise of transnational neoliberalism in the 1980s. London/New York: Routledge.

Palley, T. I. (2013): Europe's Crisis without End: The Consequences of Neoliberalism. Contributions to Political Economy, 32 (1), 29-50.

Plehwe, D./Walpen, B. (1999): Wissenschaftliche und wissenschaftspolitische Produktionsweisen im Neoliberalismus. Beiträge der Mont Pèlerin Society und marktradikaler Think Tanks zur Hegemoniegewinnung und -erhaltung. PROKLA, 29 (2), 203-235.

Plickert, P. (2008): Wandlungen des Neoliberalismus. Eine Studie zu Entwicklung und Ausstrahlung der Mont Pèlerin Society. Stuttgart: Lucius \& Lucius.

Polanyi-Levitt, K. (2014): The Power of Ideas: Keynes, Hayek, and Polanyi. International Journal of Political Economy, 41 (4), 5-15.

Pressler, F. (2013): Die erste Weltwirtschaftskrise. Eine kleine Geschichte der Großen Depression. München: C.H.Beck.

Reinhoud, J./Audier, S. (2018): The Walter Lippmann Colloquium. The Birth of Neo-Liberalism. Cham: Palgrave Macmillan.

Röpke, W. (1942/1979): Die Gesellschaftskrise der Gegenwart. Bern/Stuttgart: Paul Haupt.

Rosenberger, S./Schmid, G. (2003): Treffsicher. Sozialpolitik zwischen 2000 und 2002. In: Rosenberger S./Tálos E. (Hg.): Sozialstaat. Probleme, Herausforderungen, Perspektiven. Wien: Mandelbaum, 96-120.

Rothschild, K. W. (2009): Neoliberalism, EU and the Evaluation of Policies. Review of Political Economy, 21 (2), 213-225.
Saad-Filho, A./Johnston, D. (2005): Neoliberalism. A Critical Reader. London/Ann Arbor: Pluto Press.

Sanz Bas, D. (2011): Hayek's Critique of The General Theory: A New View of the Debate between Hayek and Keynes. Quarterly Journal of Austrian Economics, 14 (3), 288-310.

Schui, H./Blankenburg, S./Ptak, R./Bachmann, G./Kotzur, D. (1997): Wollt ihr den totalen Markt? Der Neoliberalismus und die extreme Rechte. München: Knaur.

Schulmeister, S. (2013): Realkapitalismus und Finanzkapitalismus. Zwei „Spielanordnungen“ und zwei Phasen des „langen Zyklus“. In: Kromphardt, J. (Hg.): Weiterentwicklung der Keynes'schen Theorie und empirische Analysen. Schriften der Keynes Gesellschaft. Marburg: Metropolis, $115-170$.

Senft, G. (2010): Ein krankhafter Zustand des Geldmarktes. Die Finanzkrisen von 1873 und 2007/08 im Vergleich. polylog. Zeitschrift für Interkulturelles Philosophieren, 12 (23), 69-82.

Skidelsky, R. J. A. (1992): John Maynard Keynes: The Economist as Saviour, 1920-1937. London: Macmillan.

Stigler, G. J. (1988): Memoirs of an Unregulated Economist. New York: Basic Books.

Sykes, A. (2014 [1997]): The Rise and Fall of British Liberalism. 1776-1988. London/New York: Routledge.

Tálos, E. (2005): Vom Siegeszug zum Rückzug: Sozialstaat Österreich 1945-2005. Innsbruck: Studien Verlag.

Tálos, E. (2006): Sozialpartnerschaft. Austrokorporatismus am Ende? In: Dachs, H./Gerlich, P./Gottweis, H. (Hg.): Politik in Österreich. Das Handbuch. Wien: Manz Verlag, 425-442.

Thomasberger, C. (2012): Das neoliberale Credo. Ursprünge, Entwicklung, Kritik. Marburg: Metropolis.

Trudel, D. (2017): Revisiting the Origins of Communication Research: Walter Lippmann's World War II Adventure in Propaganda and Psychological Warfare. International Journal of Communication, 11, 3721-3739.

Venugopal, R. (2015): Neoliberalism as concept. Economy and Society, 44 (2), 165-187.

Walpen, B. (2000): Von Igeln und Hasen oder: Ein Blick auf den Neoliberalismus. UTOPIE kreativ, 121/122, 10661079.

Walpen, B. (2004): Die offenen Feinde und ihre Gesellschaft. Eine hegemonietheoretische Studie zur Mont Pèlerin Society. Hamburg: VSA.

Wendet, W. R. (2016): Die Geschichte der Sozialen Arbeit 1. Die Gesellschaft vor der sozialen Frage 1750-190o. Wiesbaden: Springer.

Willke, G. (2003): Neoliberalismus. Frankfurt a. M./New York: Campus.

Wullweber, J./Graf, A./Behrens, M. (2014, Hg.): Theorien der Internationalen Politischen Ökonomie. Wiesbaden: VS Verlag für Sozialwissenschaften.

Zellenberg U. (2018): Wo ist der Neoliberalismus in Osterreich? Wirtschaftspolitische Blätter, 65 (1), 135-157. 